\title{
Study of luminosity leveling with crossing angle for polarized proton program at RHIC
}

\author{
C. Liu, I. Blackler, Y. Luo, A. Marusic, \\ M. Minty, V. Ranjbar, G. Robert-Demolaize
}

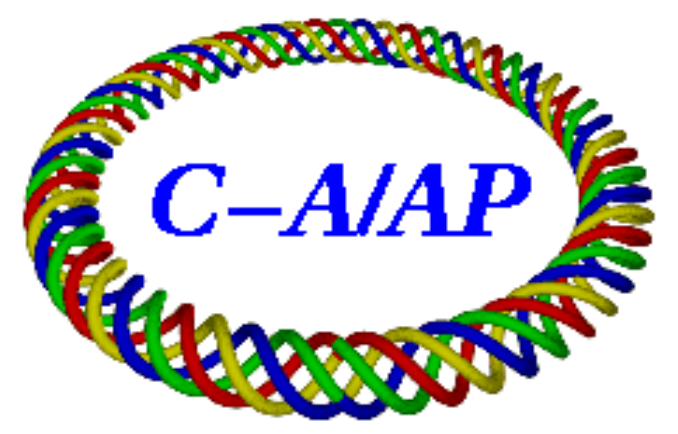

Collider-AcceleratorDepartment Brookhaven National Laboratory

Upton, NY 11973

U.S. Department of Energy

Office of Science, Office of Nuclear Physics

Notice: This document has been authorized by employees of Brookhaven Science Associates, LLC under Contract No. DE-SC0012704 with the U.S. Department of Energy. The United States Government retains a nonexclusive, paid-up, irrevocable, world-wide license to publish or reproduce the published form of this document, or allow others to do so, for United States Government purposes. 


\title{
STUDY OF LUMINOSITY LEVELING WITH CROSSING ANGLE FOR POLARIZED PROTON PROGRAM AT RHIC
}

\author{
C. Liu, I. Blackler, Y. Luo, A. Marusic, M. Minty, V. Ranjbar, G. Robert-Demolaize, \\ Brookhaven National Lab, Upton, NY, USA.
}

\section{Abstract}

Luminosity leveling has been requested by experiments in the past and it is also foreseen in the future at RHIC. There are some options to do this, some well tested and some are potential. In this report, we present the theoretical and experimental study on leveling luminosity by crossing angle.

\section{INTRODUCTION}

Luminosity leveling was requested by STAR experiment for the $250 \mathrm{GeV}$ polarized proton program in 2017. The requested luminosity averages at $1.6 \times 10^{32} \mathrm{~cm}^{-2} \mathrm{~s}^{-1}$ with less than $+/-10 \%$ fluctuation. In the past, vertical orbit offset at IRs was applied to level luminosity without issue. For polarized proton, the offset luminosity leveling was ruled out due to concern of degradation of polarization profile. The dynamic $\beta^{*}$ change during store successfully applied for Au-Au program in 2014 is one other option for luminosity leveling, which might require more development time. The PHENIX detectors were dissembled for upgrading to sPHENIX before 2017 run. The luminosity lifetime at STAR in 2017 might be improved enough with just 1 collision so that the drop of luminosity during a store is within tolerance. In parallel, we studied the possibility of luminosity leveling with crossing angle and carried out a beam experiment to test the scheme.

\section{CALCULATION OF LUMINOSITY LEVELING WITH CROSSING ANGLE}

With the assumption of Gaussian profile in three dimensions of the colliding bunches, the luminosity can be written as [1]

$$
L=\frac{f N_{1} N_{2}}{4 \pi \sigma_{0}^{2}}\left(\frac{\beta^{*}}{\sqrt{\beta_{x}^{*} \beta_{y}^{*}}}\right) \frac{1}{\sqrt{\pi} \sigma_{z}} \int_{-\infty}^{\infty} \frac{\exp \left\{-\left[\frac{\Delta_{x}+\alpha_{x} z}{2 \sigma_{x}(z)}\right]^{2}+\left[\frac{\Delta_{y}+\alpha_{y} z}{2 \sigma_{y}(z)}\right]^{2}\right\}}{\sqrt{\left[1+\left(\frac{z-z_{x 0}}{\beta_{x}^{*}}\right)^{2}\right]\left[1+\left(\frac{z-z_{y 0}}{\beta_{y}^{*}}\right)^{2}\right]}} e^{-z^{2} / \sigma_{z}^{2}} d z
$$

Where $N$ is the number of particles in a bunch, $\sigma_{0}$ is the rms beam size, $\beta^{*}$ is the beta-function at the interaction point, $\sigma_{z}$ is the rms bunch length, $\Delta$ is the offset between two beams, $\alpha$ is the full crossing angle between two beams, $z_{x 0}$ and $z_{y 0}$ are the longitudinal locations at which the minimal beta functions are in horizontal and vertical plane, and $\sigma_{x, y}(z)=\sqrt{\frac{\epsilon \beta_{x, y}^{*}}{6 \pi y}\left[1+\left(\frac{z-z_{x 0}}{\beta_{x}^{*}}\right)^{2}\right]}$. For polarized proton program, there are no transverse offsets and longitudinal shift of minimal beta functions by design, and the beams are round at the interaction point. Therefore, the formula becomes the following with only horizontal crossing angle,

$$
L=\frac{f N_{1} N_{2}}{4 \pi \sigma_{0}^{2}} \frac{1}{\sqrt{\pi} \sigma_{z}} \int_{-\infty}^{\infty} \frac{\exp \left\{-\left[\frac{\alpha_{X} z}{2 \sigma_{X}(z)^{2}}\right]^{2}\right\}}{\left[1+\left(\frac{z}{\beta^{*}}\right)^{2}\right]} e^{-z^{2} / \sigma_{Z}^{2}} d z
$$

Without crossing angle, the formula is

$$
L=\frac{f N_{1} N_{2}}{4 \pi \sigma_{0}^{2}} \frac{1}{\sqrt{\pi} \sigma_{z}} \int_{-\infty}^{\infty} \frac{e^{-z^{2} / \sigma_{Z}^{2}}}{\left[1+\left(\frac{Z}{\beta^{*}}\right)^{2}\right]} d z
$$

The luminosity scale factor due to crossing angle is the ratio between (1) and (2),

$$
R=\int_{-\infty}^{\infty} \frac{\exp \left\{-\left[\frac{\alpha_{x} z}{2 \sigma_{X}(z)}\right]^{2}\right\}}{\left[1+\left(\frac{z}{\left.\beta^{*}\right)^{2}}\right]\right.} e^{-z^{2} / \sigma_{z}^{2}} d z / \int_{-\infty}^{\infty} \frac{e^{-z^{2} / \sigma_{Z}^{2}}}{\left[1+\left(\frac{z}{\beta^{*}}\right)^{2}\right]} d z
$$

For simplicity, we could use the short bunch assumption, which is a rough approximation for polarized proton case, and then the scale factor is [2] 


$$
R=\frac{1}{\sqrt{1+\left(\alpha_{x} \sigma_{z} / 2 \sigma_{0}\right)^{2}}}
$$

We would like to know how much of an angle is required to achieve a leveled luminosity at $1.6 \times 10^{32} \mathrm{~cm}^{-2} \mathrm{~s}^{-1}$. Take a store from 2013 as an example, we will calculate the angle based on formula presented above. The luminosity and the required scale factor are showed in Fig. 1 . The calculated angle over the course of the store is shown in Fig. 2. The beam parameters used for the calculation are: $\beta^{*}=0.7 \mathrm{~m}, \epsilon_{n, 95 \%}=15 \mu \mathrm{m}, \sigma_{z}=3.4 \mathrm{~ns}$.
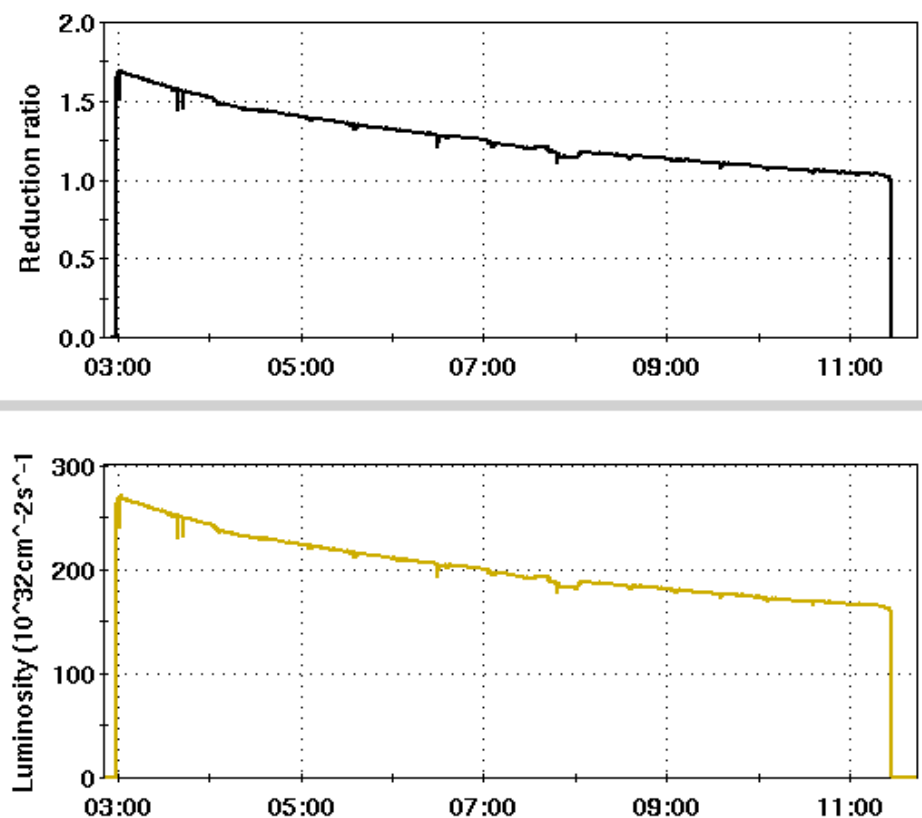

Fig. 1: The recorded luminosity during a store in 2013 in the lower plot, the required scale factor to achieve leveled luminosity at $1.6 \times 10^{32} \mathrm{~cm}^{-2} \mathrm{~s}^{-1}$ in the upper plot.

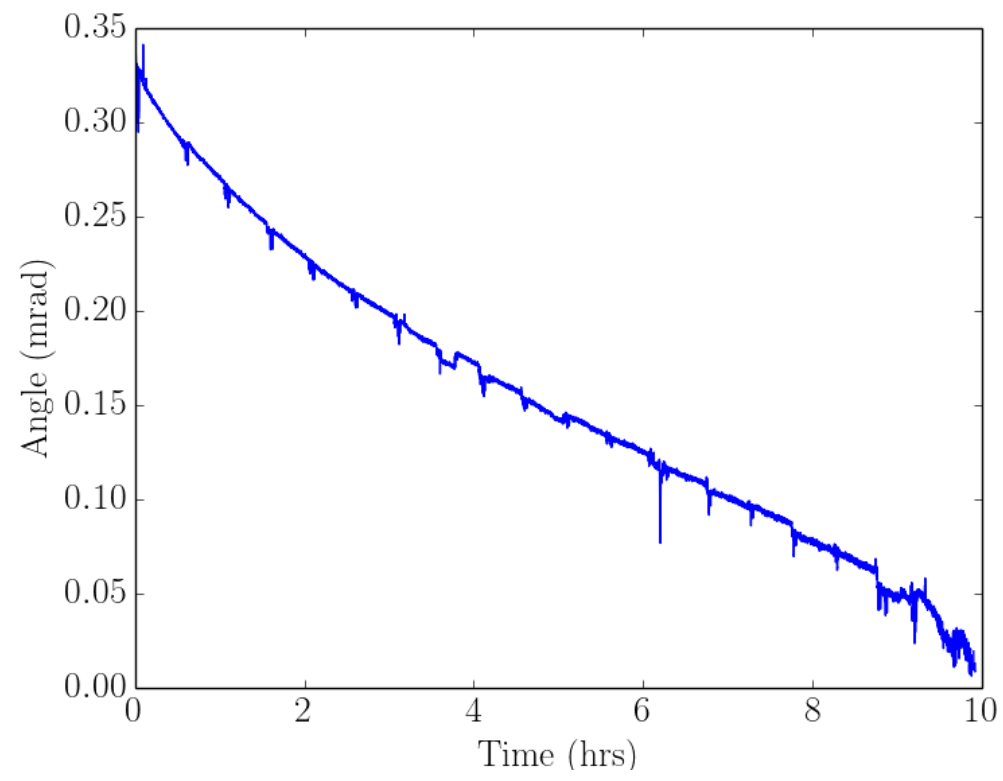

Fig. 2: The calculated crossing angle during the store needed to level the luminosity at $1.6 \times 10^{32} \mathrm{~cm}^{-2} \mathrm{~s}^{-1}$. 
As a benefit of crossing angle, the vertex distribution is narrower. The comparison of vertex with and without a crossing angle is shown in Fig. 3.

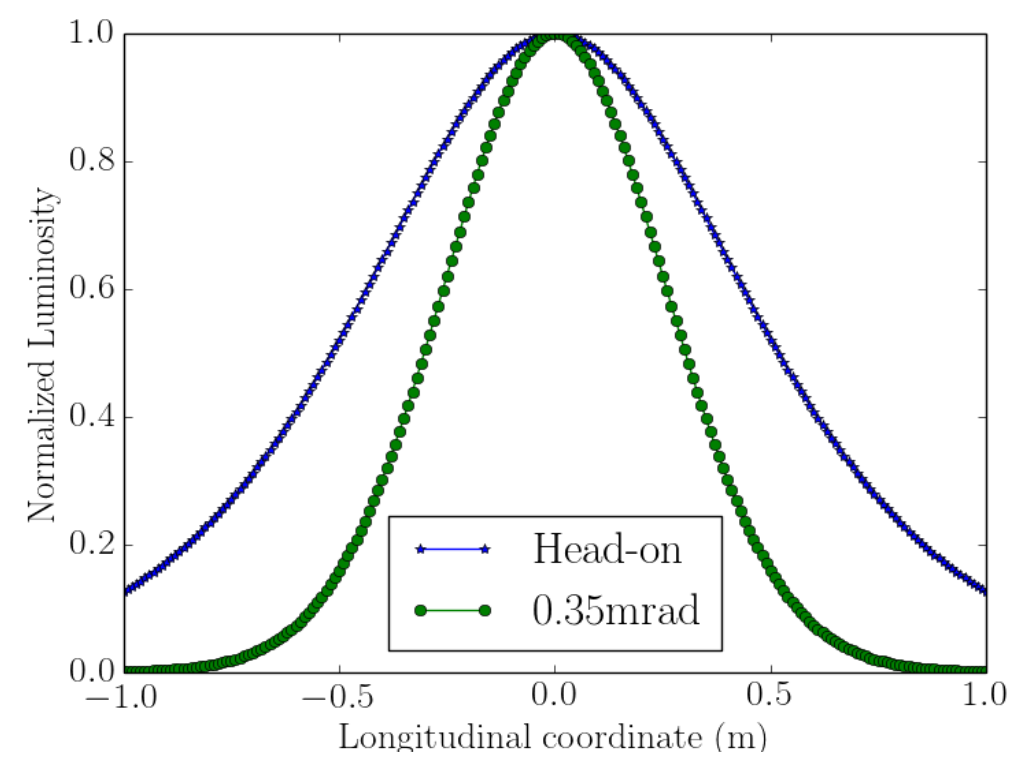

Fig. 3: The vertex distribution for head-on collision and collision with 0.35 mrad crossing angle.

\section{EXPERIMENTAL RESULTS}

During Au-Au program in 2016, we studied the luminosity leveling with crossing angle experimentally. The machine was filled with 56*56 bunches, with bunch intensity of 2E9. The re-bucketing was turned off so that the bunch length is comparable to that of proton beam. Stochastic cooling, periodical orbit feedback and leveling with offset were turned off to avoid interference with the study. The horizontal crossing angle was dialed in by DX and D0 magnets. The crossing angles dialed in at each step were confirmed by the currents of DX and D0 shunt supplies. The vertical crossing angle was set by dipole corrector magnets. The ZDC rates and the beam loss during the beam study are shown in Fig. 4. The maximum vertical crossing angle by dipole correctors is about 0.2 mrad before beam loss at triplets for Yellow beam. There was no adverse effect observed on the beam with crossing angle. This excludes the concern of synchro-betatron resonance with crossing angle.
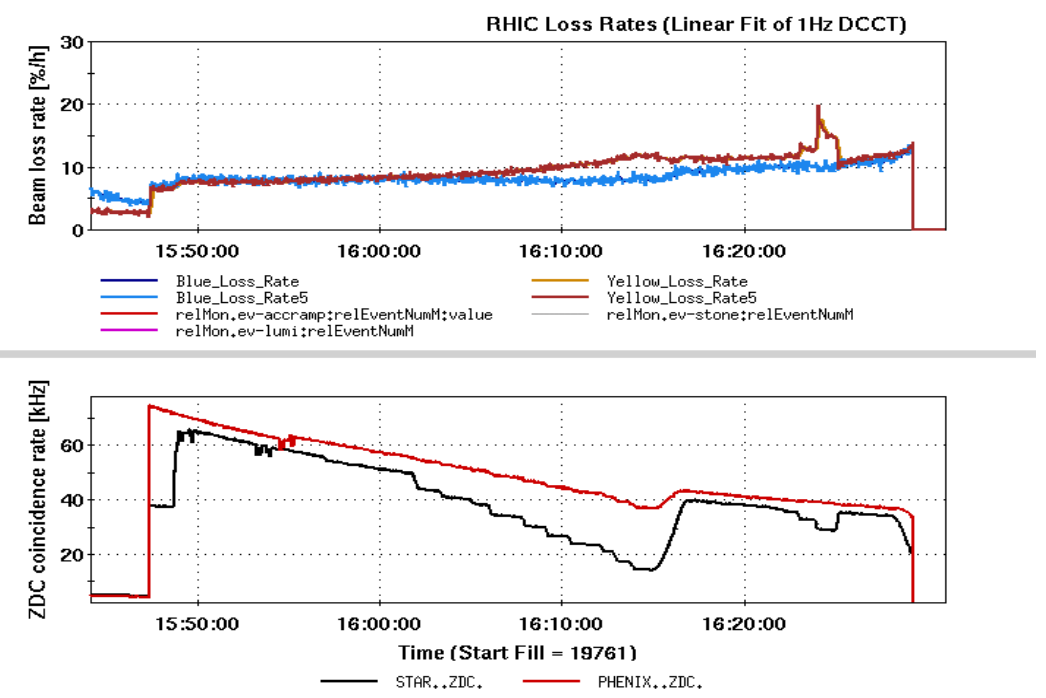

Fig. 4: The recorded ZDC rates in the lower plot and the beam loss rate in the upper plot. 
The ZDC rate at PHENIX was scaled to be the same as that at STAR at the start of the study (Fig. 5) so that we take it as the baseline rate, or the would-be rate at STAR without crossing angle. The scale factor is 0.914 .

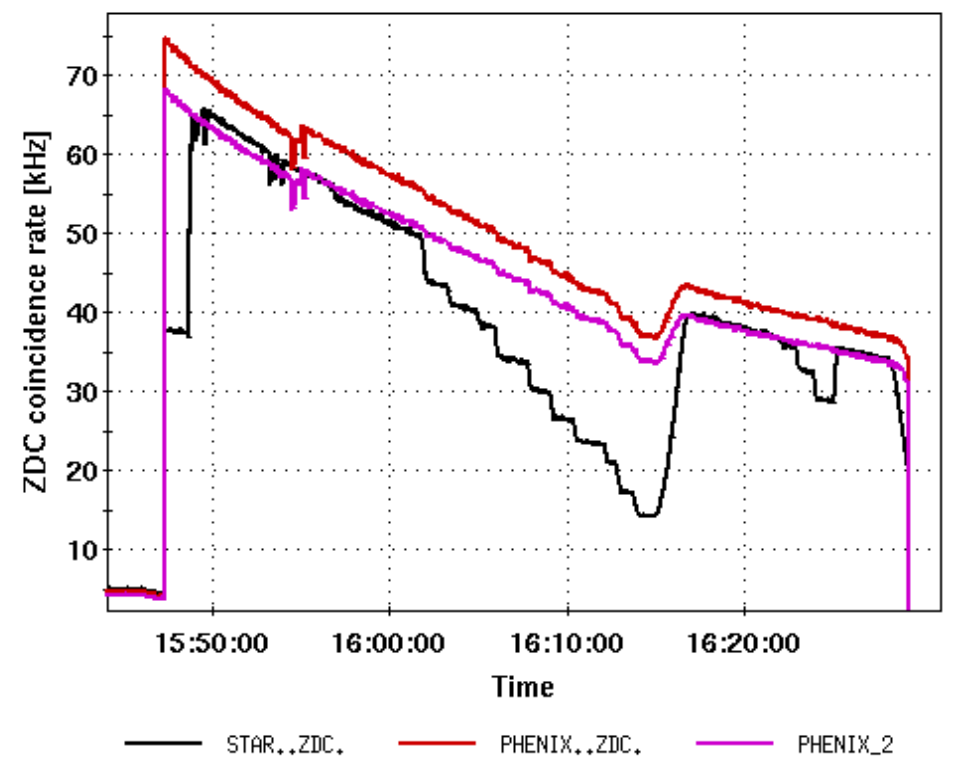

Fig. 5: The PHENIX ZDC rate was scaled by a factor of 0.914 to be used as the baseline.

The luminosity scale factor is calculated as the ratio between recorded ZDC rate at STAR and the scaled ZDC rate at PHENIX (Fig. 6).
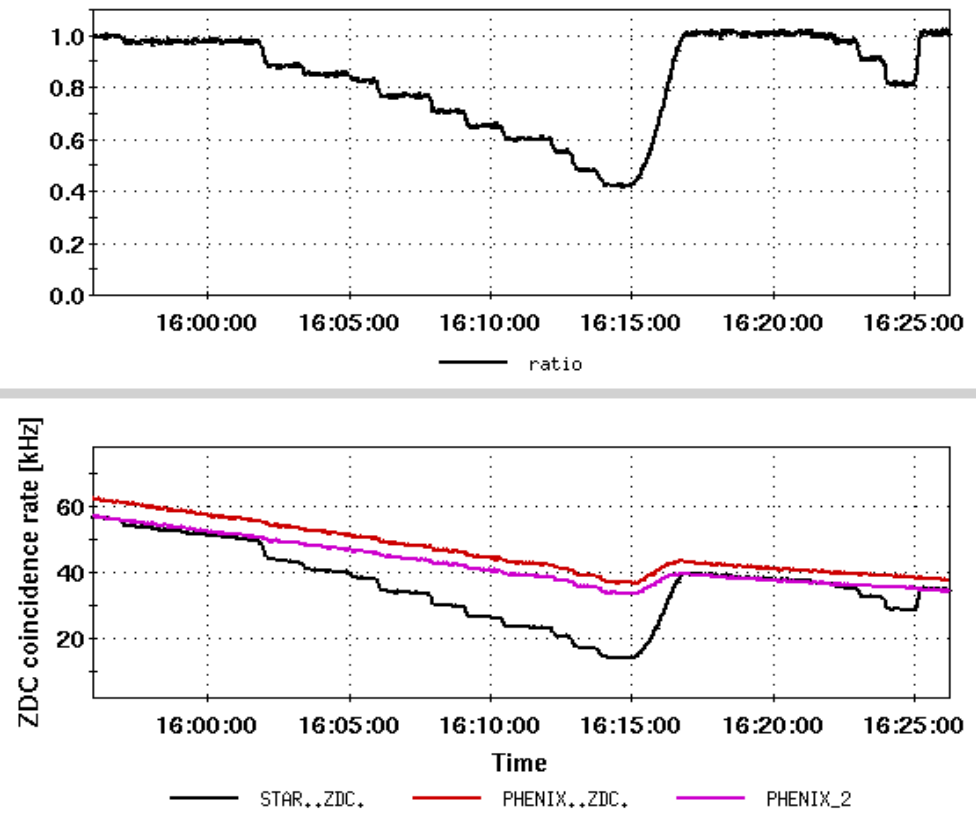

Fig. 6: The measured ZDC rate scale factor in the upper plot.

The measured ZDC rates scale factor versus crossing angle is shown in Fig. 7 in red. With single correction, the luminosity scale factor is shown in light blue. One could also fit the luminosity at STAR based on the numbers before and after the study and use it as the baseline. Then the scale factor is shown in magenta. For comparison, the calculated scale factor based on Eq. (4) is shown in solid blue, and the scale factor based on Eq. (3) is shown in solid 
green. It is expected that the agreement of Eq. (3) and the measurement data is better because there was no short bunch assumed like in Eq. (4).

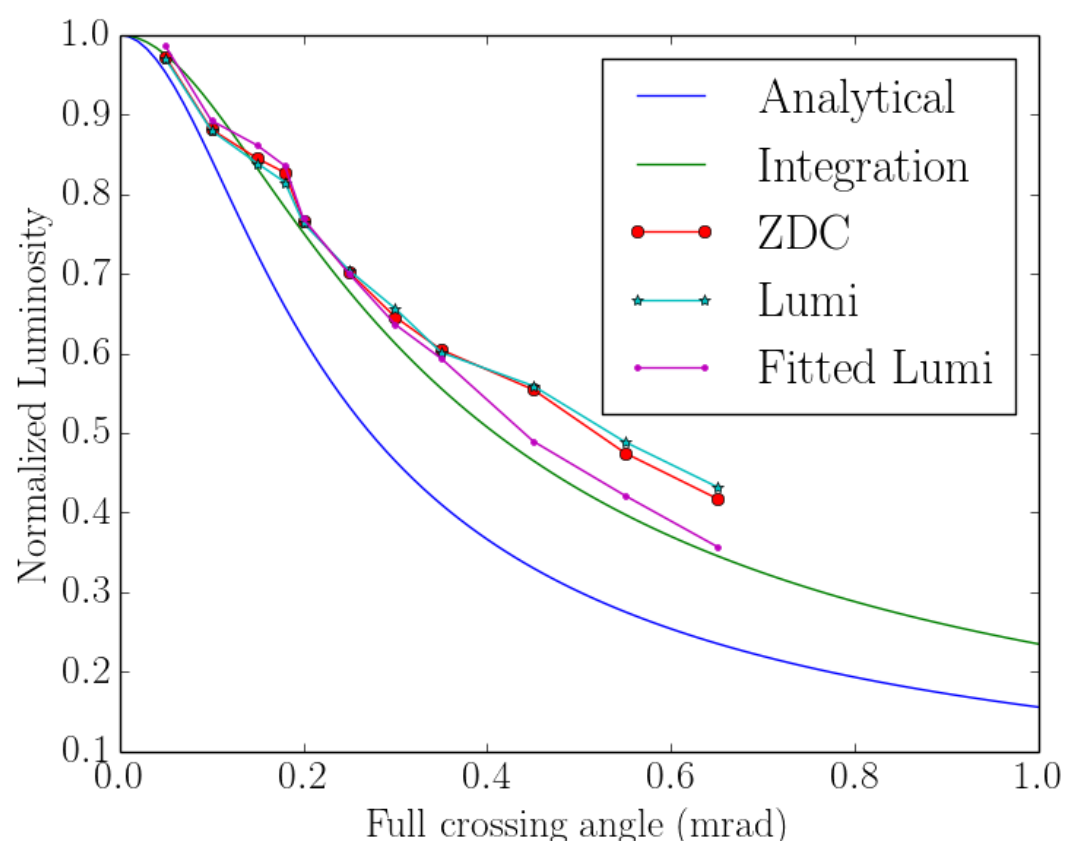

Fig. 7: The comparison of measured and calculated scale factor.

During the measurement, the transverse and longitudinal dimensions evolve with time as shown in Fig. 8. The emittance and bunch length change over time have effects on the Luminosity dependency on crossing angle.
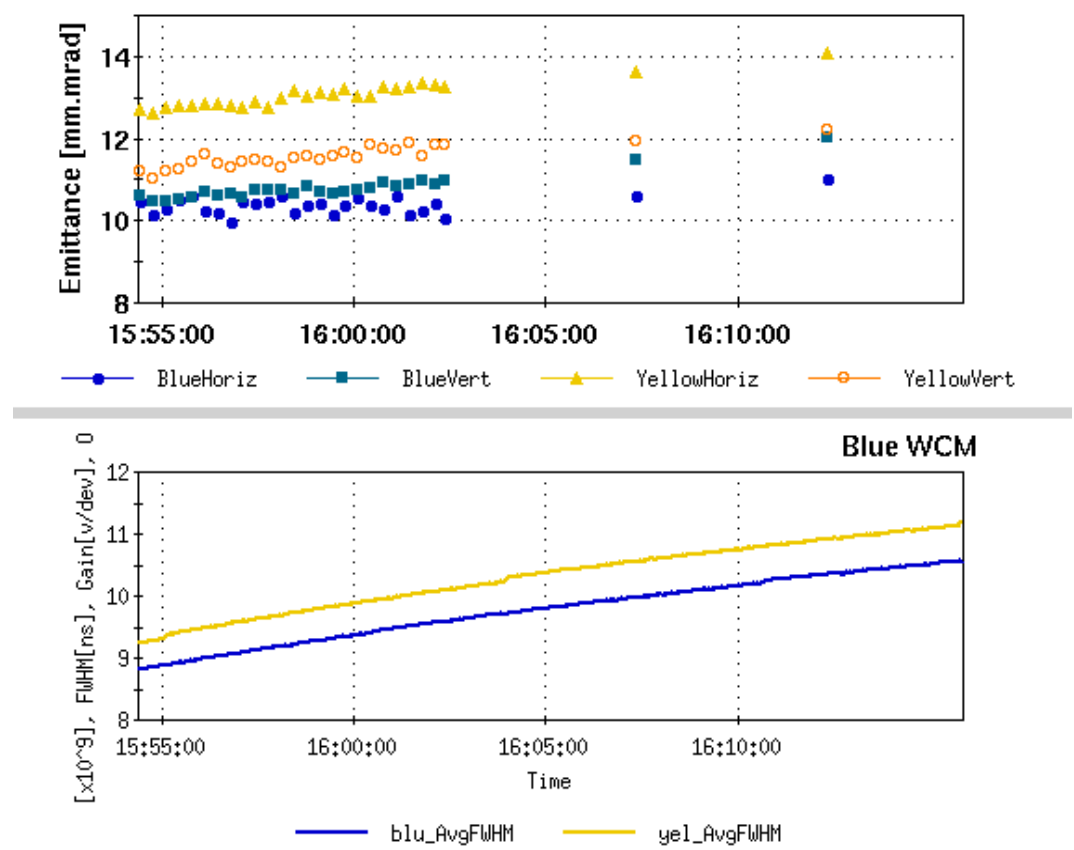

Fig. 8: The transverse emittance and bunch length evolution during the beam study. 


\section{SUMMARY}

The scheme of luminosity leveling with horizontal crossing angle by D0 and DX magnets is robust. The required crossing angle is within the tuning range, which is $3 \mathrm{mrad}$ in full angle limited by DX aperture. The experimental study confirmed theoretical calculation. There was no adverse effect on the beam due to crossing angle. Tape sequence was worked on to implement this scheme continuously during store.

\section{REFERENCE}

[1] M. Syphers, “Some notes on Luminosity Calculations”, Fermilab note, Beams-doc-1348, 2007

[2] H. Werner and B. Muratori. "Concept of luminosity." proceedings of CERN Accelerator School. Vol. 361. 2003. 